\title{
Interpretation Training in Individuals with Generalized Social Anxiety Disorder: A Randomized Controlled Trial
}

\author{
Nader Amira and Charles T. Taylor \\ Department of Psychology, San Diego State University
}

\begin{abstract}
Objective-To examine the efficacy of a multi-session computerized Interpretation Modification Program (IMP) in the treatment of Generalized Social Anxiety Disorder (GSAD).

Method-The sample comprised 49 individuals meeting diagnostic criteria for GSAD who were enrolled in a randomized, double-blind placebo-controlled trial comparing IMP $(n=23)$ to an Interpretation Control Condition (ICC; $n=26$ ). The interpretation training procedures comprised a word-sentence association task in which participants decided whether or not a word implying a threatening or benign meaning was related to an ambiguous social scenario. In the IMP group, participants were reinforced for interpreting ambiguous social information in a non-threatening and more benign manner. In the ICC group, participants were reinforced with equal frequency for interpreting ambiguous social information in either a threatening or benign manner.
\end{abstract}

Results-Intent-to-treat and completer analyses revealed that IMP significantly decreased threat interpretations and increased benign interpretations from pre- to post-assessment relative to the ICC group. Moreover, IMP participants displayed significantly larger reductions in clinician-rated social anxiety symptoms and functional impairment as well as self-reported trait anxiety and depression relative to ICC participants. Groups did not differ on change in self-rated social anxiety symptoms. Participants no longer meeting DSM-IV criteria for GSAD at post-assessment were $65 \%$ in IMP and $13 \%$ in ICC.

Conclusions-These results suggest that computerized interpretation training procedures may be beneficial for treating social anxiety disorder.

\section{Keywords}

Social anxiety disorder; Interpretation; Cognitive Bias Modification; Treatment; Information Processing

\begin{abstract}
Social Anxiety Disorder (SAD) is a common and disabling condition (e.g., Stein \& Stein, 2008 ) with a lifetime prevalence of $12 \%$ (Kessler et al., 2005). Research supports the efficacy of a number of psychosocial (e.g., Hofmann \& Smits, 2008) and pharmacological treatments for SAD (e.g., Stein \& Stein, 2008). Despite their established efficacy, however, extant treatments leave room for improvement. For example, using conventional treatment strategies (i.e., cognitive behavioral or pharmacological regimens), only about $50 \%$ of
\end{abstract}

\footnotetext{
${ }^{a}$ Corresponding author: Nader Amir, Ph.D., Department of Psychology, San Diego State University, San Diego, CA 92120, Phone: (619)229-3740, Fax: (619)229-3743, namir@mail.sdsu.edu.

The following manuscript is the final accepted manuscript. It has not been subjected to the final copyediting, fact-checking, and proofreading required for formal publication. It is not the definitive, publisher-authenticated version. The American Psychological Association and its Council of Editors disclaim any responsibility or liabilities for errors or omissions of this manuscript version, any version derived from this manuscript by $\mathrm{NIH}$, or other third parties. The published version is available at www.apa.org/pubs/journals/ccp.
} 
patients diagnosed with SAD are classified as treatment responders (e.g., Heimberg et al., 1998; Davidson et al., 2004; see Stein \& Stein, 2008 for a review). Moreover, many individuals diagnosed with $\mathrm{SAD}$ fail to access existing empirically supported treatments (Coles, Turk, Jindra, \& Heimberg, 2004; Huppert, Franklin, Foa, \& Davidson, 2003; Olfson et al., 2000). Considered together, these findings underscore the potential value of exploring novel treatment approaches that may augment existing interventions and/or increase accessibility to effective treatments for SAD.

Cognitive Bias Modification (CBM) research represents one emerging area of translational clinical science that shows promise in the development of novel interventions for emotional disorders (see Beard, 2011; Koster, Fox, \& MacLeod, 2009 for recent reviews). CBM involves the application of experimental procedures used in cognitive science to measure basic cognitive processes (e.g., attention, interpretation) to manipulate factors involved in the etiology and maintenance of emotional disorders (Mathews \& MacLeod, 2005). Previous research supports the efficacy of CBM procedures in modifying selective information processing biases for emotionally salient stimuli (e.g., Grey \& Mathews, 2000; MacLeod, Rutherford, Campbell, Ebsworthy, \& Holker, 2002) and conferring differential emotional vulnerability under conditions of heightened stress (e.g., Amir, Beard, Weber, Bomyea, \& Taylor, 2008). Although promising, the majority of previous studies have examined the effects of CBM procedures in non-clinical samples, on state measures of anxiety or depression (rather than symptoms), using brief (e.g., single-session) manipulations. Moreover, few studies have tested the clinical utility of CBM procedures in the remediation of clinical disorders and those that have done so, have been restricted to attentional manipulations (CBM-A; Amir, Beard, Burns, \& Bomyea, 2009; Amir, Beard, Taylor et al., 2009; Schmidt, Richey, Buckner, \& Timpano, 2009) or have combined attentional and interpretation manipulations (Beard, Weisberg, \& Amir, in press; Brosan, Hoppitt, Shelfer, Sillence, \& Mackintosh, 2011). Thus, our goal in the present study was to extend previous CBM research by testing the efficacy of a multi-session computerized procedure designed to modify interpretation bias alone in individuals meeting diagnostic criteria for generalized social anxiety disorder (GSAD).

Interpretation bias towards threat refers to the tendency to construe ambiguous information in an overly threatening manner. Cognitive models of emotional disorders propose that interpretation bias may be particularly influential in individuals with SAD because social cues are often ambiguous and therefore open to a range of interpretations (Clark \& Wells, 1995; Rapee \& Heimberg, 1997). For example, during a social interaction a conversation partner's laughter after a comment one makes may indicate ridicule (threat interpretation) or amusement (benign interpretation). In support of cognitive models, previous studies find that socially anxious individuals display greater endorsement of threat interpretations compared to non-anxious controls (e.g., Amir, Foa, \& Coles, 1998; Stopa \& Clark, 2000; Huppert, Foa, Furr, Filip, \& Mathews, 2003). Moreover, negative interpretation bias has been found to mediate the relationship between social anxiety and state anxiety in response to socialevaluative threat (Beard \& Amir, 2010). Research also suggests that socially anxious individuals lack the benign interpretation bias exhibited by non-anxious individuals (Constans, Penn, Ihen, \& Hope, 1999; Hirsch \& Mathews, 2000; Moser, Hajcak, Huppert, Foa, \& Simons, 2008).

Numerous studies support the efficacy of CBM procedures in modifying interpretation bias (CBM-I; see Beard, 2011 for a review). Although methodologies vary across studies, the basic premise underlying CBM-I procedures involves manipulating the frequency with which an ambiguous stimulus and a target stimulus that conveys one meaning of the ambiguous information are paired together. This manipulation is intended to encourage participants to think of the ambiguous information in either a negative or positive way. For 
example, in their seminal study, Grey and Mathews (2000) used homographs (i.e., words with multiple meanings, e.g., 'mean' can imply 'average' or 'nasty'), to modify interpretations in non-anxious individuals. In one experiment, participants saw homographs followed by target words implying a threatening ('nasty') or benign ('average') meaning of the homograph ('mean'). Participants decided whether or not the two words were related. When tested later with new homographs, participants in the threat condition were faster to respond to threat interpretations in a lexical decision task. Similarly, participants in the benign condition were faster to respond to benign interpretations.

Subsequent extensions of this research in non-anxious (e.g., Mathews \& Mackintosh, 2000; Wilson, MacLeod, Mathews, \& Rutherford, 2006) and high anxious samples (e.g., Hayes, Hirsch, Krebs \& Mathews, 2010) have demonstrated that experimentally manipulating interpretation of ambiguous information can causally influence anxiety and related outcomes (e.g., negative thought intrusions) in response to laboratory-induced stress (see, however, Salemink, E., van den Hout, M., \& Kindt, 2007). More relevant to the current study, in a sample of individuals with elevated levels of social anxiety, participants who received a single-session benign interpretation induction procedure reported experiencing less anticipatory anxiety about a future social situation relative to the control group (Murphy, Hirsch, Mathews, Smith, \& Clark, 2007). To our knowledge, four published studies have examined the effects of multi-session computerized interpretation training programs in reducing anxiety symptoms. First, in a sample of individuals with high levels of trait anxiety, Mathews, Ridgeway, Cook, and Yiend (2007) demonstrated that a four-session interpretation training procedure (administered over one week) that encouraged participants to resolve ambiguous scenarios in a positive manner resulted in significantly lower trait anxiety scores at post-assessment relative to a control group who did not receive the training procedure. Similarly, Salemink, van den Hout, and Kindt (2009) found that high trait anxious students who completed a positive interpretation training program online for eight consecutive days were less state and trait anxious, and scored lower on a measure of general psychopathology compared to the control group. However, no effects were observed on measures of social anxiety and stress vulnerability.

Most relevant to the current study, Beard and Amir (2008) examined the effects of an eightsession interpretation training program in an analogue sample of 27 individuals with elevated levels of self-reported social anxiety. Using a modified word-sentence association paradigm (WSAP; Beard \& Amir, 2009), participants in the two experimental conditions were differentially reinforced for rejecting threatening interpretations and endorsing benign interpretations of ambiguous social information. Results revealed that participants who were trained to repeatedly interpret ambiguous social information in a less threatening and more benign manner displayed a significant reduction in threat interpretations and increase in benign interpretations. Moreover, the actively trained group displayed a significantly larger reduction in social anxiety symptoms compared to the control condition. Change in benign interpretations mediated the relationship between interpretation training and change in social anxiety. The groups did not differ, however, on change in trait anxiety or depression. More recently, two studies have found that a combined regimen that included the interpretation procedure used by Beard and Amir (2008) and an attentional training procedure (Amir, Beard, Taylor et al., 2009; MacLeod et al., 2002) resulted in significant reductions in anxiety symptoms in individuals diagnosed with GSAD (Beard et al., in press) or either GSAD or GAD (Brosan et al., 2011).

In summary, previous research supports the efficacy of CBM procedures in modifying interpretations of ambiguous information and reducing anxiety symptoms in analogue and clinical samples. However, previous CBM-I studies were conducted in small, analogue samples (Beard \& Amir, 2008) or in combination with CBM-A procedures (Beard et al., in 
press; Brosan et al., 2011). Thus, the efficacy of interpretation training procedures alone in individuals presenting with greater severity of symptoms and functional interference remains to be established. Accordingly, the primary aim of the current study was to test the efficacy of a multi-session Interpretation Modification Program (IMP) in a sample of patients meeting diagnostic criteria for GSAD. We predicted that IMP would reduce threat interpretations, increase benign interpretations, as well as decrease social anxiety symptoms and associated functional impairment from pre- to post-assessment relative to the Interpretation Control Condition (ICC). We also explored the effects of IMP on symptoms of trait anxiety and depression.

\section{Method \\ Design}

The current study comprised a 2 (Group: IMP, ICC) X 2 (Time: pre-assessment, postassessment) mixed design. Participants were randomly assigned to IMP $(n=23)$ or ICC $(n=$ 26). They were assessed using interviewer, self-report, and information processing bias measures at pre- and post-assessment. Follow-up assessments were conducted on participants in the IMP group approximately three months after the post-assessment.

\section{Participants}

Participants were recruited through clinical referrals as well as posted announcements in community settings and local newspapers that described the program and provided a telephone contact number. Diagnostic assessment was based on an initial telephone screening followed by an in-person diagnostic interview using the Structured Clinical Interview for the DSM-IV (SCID; First, Spitzer, Gibbon, \& Williams, 1994). We administered the full SAD module of the SCID as well as the SCID screening module to screen for co-occurring Axis I conditions. Of the 62 individuals who were assessed for eligibility, 11 did not meet entry criteria and 2 were lost to contact. The remaining 49 participants met a principal DSM-IV diagnosis of GSAD. Exclusionary criteria included: (a) evidence of suicidal intent, (b) evidence of current substance abuse or dependence, (c) evidence of current or past schizophrenia, bipolar disorder, or organic mental disorder, (d) any concurrent psychotherapy (e) change in pharmacological treatments during the 12 weeks prior to study entry, (f) cognitive behavioral therapy within the past 6 months, and (g) Liebowitz Social Anxiety Scale (LSAS) $<50 .{ }^{1}$ Participants were re-assessed using the SCID-SAD module at post- and follow-up assessments. Participants' progress through the study is summarized in Figure 1.

Eligible participants were randomly assigned to receive either the IMP or ICC. Participants were enrolled in the study serially at two sites, the University of Georgia (UGA, $n=10$ ) and San Diego State University (SDSU, $n=39$ ), between October 2004 and March 2006, August 2007 and July 2010, respectively. Participants enrolled at UGA completed an eight-session version of the treatment procedures that were otherwise identical to the 12-session program. 2 Participants randomized to the IMP versus ICC conditions at the two sites did not differ, [UGA: IMP, $n=6$ vs. ICC, $n=4$; SDSU: IMP, $n=17$ vs. ICC, $n=22$ ], $\chi^{2}(1)=.86, p=.35$. Six participants withdrew from the study prematurely (IMP, $n=3$; ICC, $n=3$ ). Reasons

\footnotetext{
${ }^{1}$ Some research recommends using an LSAS cutoff of 60 in addition to a diagnostic assessment to establish the generalized subtype of social anxiety disorder (Mennin et al., 2002). To facilitate comparability of the current findings with previous clinical trials that used this LSAS cutoff as part of their inclusion criteria, we re-analyzed our primary outcome measures (LSAS, SPAI) in the subgroup of participants with a pre-assessment LSAS $\geq 60$ (IMP, $n=16$; ICC, $n=18$ ). Results of the Group (IMP, ICC) X Time (pre-, postassessment) MANOVA revealed a significant interaction, $F(2,31)=8.29, p=.001, \eta_{p}^{2}=.35$. Follow-up univariate ANOVAs revealed a significant Group X Time interaction for the LSAS, $F(1,32)=12.78, p=.001, \eta_{p}^{2}=.29$, but not SPAI, $F(1,32)=0.059, p$ $=.81, \eta_{p}{ }^{2}=.00$. These findings are consistent with the results reported for the overall sample.
} 
provided for discontinuing the study were: pursuing other treatment $(n=1)$, relocation $(n=$ $1)$, and unknown $(n=4)$. Thus, the treatment completer sample comprised 20 IMP and 23

ICC participants. See Table 1 for demographic and clinical characteristics.

\section{Measures}

We used a battery of clinician- and self-rated measures at pre- and post-assessment. Clinician ratings were made by raters blind to treatment condition. Interviewers were doctoral level graduate students, postdoctoral fellows, and the principal investigator. Reliability training procedures followed standardized protocols used in previous clinical trials shown to maintain high inter-rater agreement (see Amir, Beard, Taylor et al., 2009 for details).

Social anxiety symptoms-Our primary outcome measure was the clinicianadministered Liebowitz Social Anxiety Scale (LSAS; Liebowitz, 1987), a 24-item scale that provides separate scores for fear and avoidance of social interaction and performance situations. The LSAS has strong psychometric properties (Heimberg et al., 1999) and is arguably the gold-standard outcome measure in treatment research in SAD (e.g., Clark et al., 2006; Heimberg et al., 1998). Our secondary outcome assessment of social anxiety symptoms was the Social Phobia and Anxiety Inventory (SPAI; Turner, Beidel, Dancu, \& Stanley, 1989), a 45-item self-rated measure that assesses the cognitive, behavioral and somatic dimensions of SAD. Previous research suggests that the SPAI has sound psychometric properties (e.g., Turner et al., 1989). Internal consistencies for these measures in the current sample were satisfactory (Average Cronbach's alpha $=.96$ and .98 , for the LSAS and SPAI, respectively).

Functional impairment-To assess current level of interference due to social anxiety, independent assessors administered the Sheehan Disability Scale (SDS; Leon, Olfson, Portera, Faber, \& Sheehan, 1997), a three-item measure that assesses functional impairment across several domains (social, work/school, family). The SDS generally demonstrates satisfactory reliability and correlates highly with other commonly used disability measures in social anxiety (e.g., Hambrick, Turk, Heimberg, Schneier, \& Liebowitz, 2004; Leon et al., 1997). Average internal consistency of the SDS in the current sample was acceptable $($ Cronbach's alpha $=.79)$.

Emotional distress-We used the State Trait Anxiety Inventory-Trait (STAI-T; Spielberger, Gorsuch, Lushene, Vagg, \& Jacobs, 1983) to assess anxious mood, and the Beck Depression Inventory II (BDI-II; Beck, Steer, \& Brown, 1996) to assess depressed mood. Average Cronbach's alphas in the current sample were .93 and .94 for the STAI-Trait and BDI-II, respectively.

\section{Computerized Interpretation Assessment}

To assess the effect of training on participants' interpretation of ambiguous social information, participants completed a computerized interpretation assessment at pre- and post-assessment called the Word Sentence Association Paradigm (WSAP; Beard \& Amir, 2009). Each WSAP trial comprised four phases. First, a fixation cross appeared on the computer screen for $500 \mathrm{~ms}$. The fixation cross directed the participants' attention toward the middle of the screen and alerted them that a trial was beginning. Second, a word

\footnotetext{
${ }^{2}$ Participants enrolled at UGA completed an eight-session version of the treatment procedures that were otherwise identical to the 12session program. We reanalyzed the data to examine whether treatment site moderated the main outcomes using a series of Group (IMP, ICC) X Site (UGA, SDSU) X Time (pre-, post-assessment) MANOVAs with repeated measurement on the last factor. Results revealed that treatment site did not significantly moderate any of the main outcomes (all $p>.10$ ).
} 
representing either a threat interpretation (e.g., "embarrassing") or a benign interpretation (e.g., "funny") appeared in the center of the computer screen for $500 \mathrm{~ms}$. Third, an ambiguous sentence (e.g., "People laugh after something you said") appeared and remained on the screen until participants pressed the space bar indicating that they finished reading the sentence. Finally, the computer prompted participants to press '\#1' on the number pad if they thought the word and sentence were related or to press ' \#3' on the number pad if the word and sentence were not related. The next trial began immediately after the participants' response.

Participants completed 110 trials during each assessment: 76 trials related to ambiguous social situations (e.g., You make a first impression on your new coworker) and 34 filler trials related to general (non-social) ambiguous situations. (e.g., The doctor examined your growth). We used two sets of WSAP materials (Set A and B) that were matched with respect to the type of situation depicted (see Appendix for sample materials). To examine whether changes in cognitive bias following interpretation training would generalize to novel stimuli, we used materials in the pre- and post-assessment blocks that were not encountered during training. After random assignment, 23 participants received Set A (IMP, $n=11$; ICC, $n=$ 12), and 26 participants received Set B (IMP, $n=12$; ICC, $n=14$ ) at pre-assessment, $\chi^{2}(1)=.014, p=.91 .^{3}, 4$

All text appeared in black, 12 point font against a gray background. For each participant, we calculated the percentage of threatening interpretations and benign interpretations endorsed. Previous research demonstrates that socially anxious individuals tend to endorse more threat-related interpretations and fewer benign interpretations of ambiguous social material relative to non-anxious controls (Beard \& Amir, 2009). Moreover, severity of social anxiety symptoms has been shown to significantly correlate with both threat and benign endorsement rates.

\section{Procedure}

Participants provided written informed consent at the beginning of the pre-assessment, and again before each session. The consent form stated that the purpose of the study was to "evaluate the usefulness of new computer based treatments for anxiety"; however, no information was provided regarding the rationale for either of the two conditions. Participants were also informed that they would be "randomly assigned to one of two groups: One group will receive the anxiety treatments and the other group will receive the non-treatment (placebo) condition". Participants completed a baseline assessment that comprised the interviewer and self-report measures. The pre-assessment, as well as postand follow-up assessments lasted approximately $2-3$ hours.

Following the pre-assessment, participants were randomly assigned to one of two conditions: IMP or ICC. Condition assignment was determined using a random number generator. A research coordinator then placed the randomly assigned condition number in separate envelopes for each participant at the beginning of the study. Prior to each experimental session, participants entered the number in their file into the computer, which began the appropriate program. Participants did not know which condition the numbers represented, and the research assistants working with the participants could not see the

\footnotetext{
${ }^{3}$ To examine whether treatment effects differed as a result of the materials used during training, we reanalyzed the data using a series of Group (IMP, ICC) X Materials Set (A, B) X Time (pre-, post-assessment) MANOVAs with repeated measurement on the last factor. Results revealed that materials set did not significantly influence any of the treatment outcomes reported in the main text (all $p$ $>.05)$.

${ }^{4}$ Data was missing for three participants; therefore, sample sizes for this analysis were, IMP $(n=18)$ and ICC $(n=22)$. We reanalyzed the data in the sub-sample of participants who had complete interpretation bias assessment data. Results of these analyses did not differ from those reported for the entire sample.
} 
number in the envelope, nor were they aware of which condition the numbers represented. Thus, participants, experimenters, and interviewers remained blind to the participant's condition until after all post-treatment assessments were conducted. The protocol included 12 20-minute interpretation training sessions delivered over a six week period (i.e., twice weekly sessions). ${ }^{2}$

After completing 12 sessions of the IMP or ICC, participants completed a post-assessment identical to the pre-assessment. Finally, to establish the duration of treatment effects, followup assessments were conducted on participants in the IMP group, which occurred approximately three months later. Participants completed the same interviewer and selfreport assessments as during the pre- and post-assessments; however, they did not complete the computerized interpretation assessment. Participants in the ICC group were offered treatment following the post-assessment. All participants received $\$ 20$ per hour as compensation for their participation (e.g., for time away from work and expenses incurred). The current procedures were approved by the Internal Review Board (IRB) at both institutions.

\section{Treatment Conditions}

Interpretation Modification Program (IMP)—The IMP procedure was identical to the WSAP, except that participants received feedback about their responses. Specifically, after participants responded regarding the relatedness of the word and sentence, the computer provided feedback about their response. Participants received positive feedback ("You are correct!') when they responded to benign interpretation trials by pressing \#1 ('related') and to threat interpretation trials by pressing \#3 ('not related'). Thus, participants received positive feedback when they endorsed benign interpretations or rejected threat interpretations of the ambiguous sentences on $100 \%$ of trials. Participants received negative feedback ("You are incorrect.") when they responded to threat interpretation trials by pressing \#1 ('related') and to benign interpretation trials by pressing \#3 ('not related'). Thus, they received negative feedback when they endorsed threat interpretations or rejected benign interpretations on $100 \%$ of trials. This feedback manipulation was intended to reinforce a benign interpretation bias and extinguish the threat interpretation bias. Participants completed two blocks of 110 training trials (76 social and 34 non-social) in each session. Participants who completed Set A during the WSAP assessment saw Set B during the IMP and vice versa. Thus, participants were assessed with different materials than those seen during the IMP. Each IMP session lasted approximately 20 minutes.

Interpretation Control Condition (ICC) - The ICC was identical to the IMP except that participants received positive feedback when they endorsed threat interpretations on half $(50 \%)$ of the trials and negative feedback when they endorsed threat interpretations for the remaining half $(50 \%)$ of trials. This frequency was the same for benign interpretations. Thus, the control group was reinforced equally for making threat and benign interpretations. The ICC was not intended to change interpretation significantly in either direction.

\section{Statistical Analyses}

Based on prior research examining the effect of a similar multi-session interpretation training procedure on symptoms of social anxiety (Beard \& Amir, 2008), we estimated an average between-group controlled effect size of 1.0 on our primary dependent measures. With alpha set at .05 and power (1-beta) set at .80, a sample size of at least 17 participants per group was needed to detect an effect of this magnitude between the IMP and ICC groups on the primary outcome measures of social anxiety symptoms. 
Analyses were conducted on treatment completers (IMP, $n=20$; ICC, $n=23$ ) and the intentto-treat (ITT) sample (IMP, $n=23$; ICC, $n=26$ ). In the ITT sample, we employed the last observation carried forward procedure to estimate post-treatment scores in non-completers. To ensure that random assignment did not create groups differing in demographic characteristics or symptom severity, we conducted chi-square tests for categorical variables and $t$-tests for continuous variables comparing groups at pre-treatment on each demographic and dependent measure. To examine the effect of the interpretation training procedure on the dependent variables, we submitted participants' scores on the measures of interpretation bias as well as self-and interviewer-rated measures of symptoms to a series of 2 (Group: IMP, ICC) X 2 (Time: pre-, post-assessment) ANOVAs with repeated measurement on the second factor. Multivariate ANOVAs (MANOVAs) were used for conceptually related measures (i.e., interpretation bias: threat, benign; social anxiety symptoms: LSAS, SPAI; emotional distress: STAI-T, BDI-II), and a univariate ANOVA was used for functional impairment (i.e., SDS). Significant multivariate effects were followed up with corresponding univariate tests. We followed up significant interactions with within-group simple effects analyses (paired $t$-tests) as well as analyses of covariance (ANCOVA) on post-treatment scores covarying pre-treatment scores to determine whether the IMP resulted in a significant change on the relevant dependent measures from pre-to post-assessment (see Clark et al., 2006; Heimberg et al., 1998). The magnitude of symptom change was established by calculating (a) within-group effect sizes $=$ (pre-assessment mean minus post-assessment mean)/pre-assessment standard deviation, and (b) between-group controlled effect sizes = (post-assessment ICC covariance adjusted mean minus post-assessment IMP covariance adjusted mean)/pooled standard deviation.

\section{Results}

\section{Preliminary Analyses}

Comparison of Treatment Conditions at Baseline-The IMP and ICC groups did not differ on any demographic or clinical characteristics $(p s>.10)$. See Table 1 . Groups did not significantly differ in level of social anxiety, depression, trait anxiety, threat interpretation endorsement, or benign interpretation endorsement at pre-assessment ( $p s>$. 10). The means and standard deviations of these measures are presented in Table 2.

Comparison of Treatment Sites-A comparison of demographic characteristics between sites (UGA vs. SDSU) revealed significant site differences on age, $t(47)=-2.09, p$ $=.042$ and gender, $\chi^{2}(1)=5.03, p=.025$, but not for education, $t(47)=-.26, p=.80$ or ethnicity (Caucasian vs. non-Caucasian), $\chi^{2}(1)=.33, p=.57$. Participants from UGA tended to be younger and female relative to participants at SDSU.

Comparison of Treatment Completers and Non-completers-Three IMP (13.0\%) and three ICC (11.5\%) participants discontinued treatment prematurely. Groups did not differ in the proportion of non-completers, $\chi^{2}(1)=.026, p=.87$. A comparison of treatment completers and non-completers revealed that there were no significant between-group differences on any of the demographic or clinical characteristics, or on the dependent measures at baseline, all $p>.10$.

\section{Main Treatment Effects: Completer Sample}

Change in Interpretation Bias-Table 2 presents the means, standard deviations, and the results of the multivariate and univariate Group (IMP, ICC) X Time (pre-, postassessment) ANOVAs for the WSAP for treatment completers. The MANOVA for social interpretation bias revealed that the predicted multivariate Group X Time interaction was significant $(p=.001)$. Univariate ANOVAs revealed significant Group X Time interactions 
on threat interpretations $(p=.006)$ and benign interpretations $(p<.001)$. Follow-up paired $t$ tests conducted within each group revealed that participants in the IMP group showed a significant decrease in endorsement of threat interpretations from pre- to post-assessment, $[t(17)=4.91, p<.001]$ and a significant increase in endorsement of benign interpretations, $[t(17)=-5.29, p<.001]$. Similar analyses in the ICC group revealed a significant decrease in threat bias scores from pre- to post-assessment, $[t(21)=2.91, p=.008]$ and a marginally significant increase in benign interpretations, $[t(21)=-1.83, p=.082]$. An ANCOVA conducted on post-treatment scores covarying pre-treatment scores revealed that the IMP group endorsed significantly fewer social threat interpretations, $[F(1,37)=7.78, p=.008$, $\left.\eta_{p}{ }^{2}=.17\right]$ and more benign interpretations, $\left[F(1,37)=17.61, p<.001, \eta_{p}{ }^{2}=.32\right]$ relative to the ICC group at post-assessment. See Table 3 for Cohen's $d$ effect size estimates.

A similar analysis for general (non-social) interpretation bias also revealed a significant multivariate Group X Time interaction $(p=.001)$. Univariate ANOVAs revealed significant Group X Time interactions on threat interpretations $(p=.002)$ and benign interpretations $(p$ $=.012$ ). Follow-up paired $t$-tests conducted within each group revealed that participants in the IMP group displayed a significant decrease in endorsement of non-social threat interpretations from pre- to post-assessment, $[t(17)=5.15, p<.001]$ and a significant increase in endorsement of benign interpretations, $[t(17)=-5.59, p<.001]$. The ICC group exhibited a significant decrease in non-social threat interpretations from pre- to postassessment, $[t(21)=2.79, p=.011]$; however, they did not differ in benign interpretations from pre- to post-assessment, $[t(21)=-.50, p=.62]$. An ANCOVA conducted on posttreatment scores covarying pre-treatment scores revealed that the IMP group endorsed significantly fewer non-social threat interpretations, $\left[F(1,37)=16.43, p<.001, \eta_{p}^{2}=.31\right]$ and more benign interpretations, $\left[F(1,37)=14.83, p<.001, \eta_{p}{ }^{2}=.29\right]$ relative to the ICC group at post-assessment. See Table 3 for Cohen's $d$ effect size estimates.

Change in Social Anxiety Symptoms-Table 2 presents the means, standard deviations, and the results of the multivariate and univariate Group (IMP, ICC) X Time (pre-, post-assessment) ANOVAs of the SP measures. Results revealed a significant multivariate Group X Time interaction $(p=.001)$. Univariate ANOVAs revealed a significant Group X Time interaction on the LSAS $(p=.001)$, but not SPAI $(p=.57)$. Follow-up paired $t$-tests conducted within each group revealed that participants in the IMP group showed a significant decrease in scores from pre- to post-assessment on the LSAS, [ $t$ $(19)=6.45, p<.001]$ and SPAI, $[t(19)=5.82, p<.001]$. Similar analyses in the ICC group also revealed a significant decrease in scores from pre- to post-assessment on the LSAS, [ $t$ $(22)=2.88, p=.009]$ and SPAI, $[t(22)=4.25, p<.001]$. An ANCOVA conducted on postassessment scores covarying pre-assessment scores revealed that the IMP group was rated by clinicians as being less socially anxious following treatment [LSAS: $F(1,40)=11.61, p$ $\left.=.002, \eta_{p}{ }^{2}=.23\right]$. However, the groups did not differ at post-assessment on self-rated symptoms of social anxiety when controlling for baseline scores, [SPAI: $F(1,40)=.30, p=$. $\left.58, \eta_{p}^{2}=.01\right]$. See Table 3 for Cohen's $d$ effect size estimates.

Change in Functional Impairment-Table 2 presents the means, standard deviations, and the results of the Group (IMP, ICC) X Time (pre-, post-assessment) ANOVA for clinician-rated functional impairment (i.e., SDS). Results revealed significant main effects for Group and Time (both $p<.05$ ) that were qualified by a significant Group X Time interaction $(p=.041)$. Follow-up paired $t$-tests conducted within each group revealed that participants in the IMP group showed a significant decrease from pre- to post-assessment in functional impairment, [SDS: $t(18)=5.47, p<.001$ ]. Similar analyses in the ICC group revealed a significant decrease in functional impairment from pre- to post-assessment, [SDS: $t(22)=3.59, p=.002]$. An ANCOVA conducted on post-treatment scores covarying pretreatment scores revealed that the IMP group displayed significantly less functional 
impairment following treatment [SDS: $F(1,39)=6.64, p=.014, \eta_{p}^{2}=.15$ ] compared to the ICC group. See Table 3 for Cohen's $d$ effect size estimates.

Change in Emotional Distress-Table 2 presents the means, standard deviations, and the results of the multivariate and univariate Group (IMP, ICC) X Time (pre-, postassessment) ANOVAs for measures of general emotional distress. Results revealed a significant multivariate main effect for Time $(p<.001)$ that was qualified by a significant Group X Time interaction $(p=.017)$. Follow-up univariate ANOVAs revealed significant Group X Time interactions on the BDI-II $(p=.005)$ and STAI-T $(p=.043)$. Follow-up paired $t$-tests conducted within each group revealed that participants in the IMP group showed a significant decrease from pre- to post-assessment in symptoms of general anxiety, [STAI-T: $t(19)=5.48, p<.001]$ and depression, [BDI-II: $t(19)=7.07, p<.001]$. Similar analyses in the ICC group revealed a significant decrease in symptoms of general anxiety from pre- to post-assessment, [STAI-T: $t(22)=2.55, p=.018$ ] and depression, [BDI-II: $t(22)=2.51, p=.02]$. An ANCOVA conducted on post-treatment scores covarying pretreatment scores revealed that the IMP group displayed significantly less trait anxiety [STAI-T: $F(1,40)=6.83, p=.013, \eta_{p}^{2}=.15$ ] and less depression [BDI-II: $F(1,40)=7.16$, $\left.p=.011, \eta_{p}{ }^{2}=.15\right]$ compared to the ICC group. See Table 3 for Cohen's $d$ effect size estimates.

\section{Main Treatment Effects: Intent-to-Treat Sample}

We repeated the main analyses in the ITT sample. See Table 4. Results were consistent with the completer sample with the following two exceptions: First, the univariate Group X Time interaction became marginally significant for the SDS ( $p=.063)$; however, an ANCOVA conducted on post-assessment SDS scores while covarying out pre-assessment scores revealed that the IMP group was rated as less functionally impaired at post-assessment relative to the ICC group, $\left[F(1,45)=4.51, p=.039, \eta_{p}{ }^{2}=.09\right]$. Second, the univariate Group X Time interaction was no longer significant for the STAI-T $(p=.16)$ and the ANCOVA conducted on post-assessment STAI-T scores while covarying pre-assessment scores was only marginally significant, $\left[F(1,46)=3.13, p=.084, \eta_{p}^{2}=.06\right]$.

\section{Maintenance of Treatment Gains}

To explore the longer-term effects of IMP, we collected follow-up data approximately three months following the post-assessment on participants in the IMP group $(n=15 ; 75 \%$ follow-up retention rate). See Table 2. Participants and clinical evaluators were no longer blind to condition assignment at follow-up. A comparison of treatment completers who participated in the follow-up assessment versus those who did not revealed that there were no significant between-group differences on any of the demographic or clinical characteristics, or on the dependent measures at baseline or post-assessment (all $p>.05$ ) with the following two exceptions: Participants who completed the follow-up assessment were older, $[t(17.68)=-2.67, p=.016]$ and displayed higher depression scores at postassessment, [BDI-II: $t(16.41)=-2.95, p=.009]$.

A repeated measures (Time: pre, post, follow-up) MANOVA conducted on the social anxiety scales revealed a significant main effect for Time, $\left[F(4,11)=7.12, p=.004, \eta_{p}^{2}=\right.$. 72]. Simple follow-up contrasts indicated that IMP participants who provided follow-up data displayed significant reductions on the LSAS $[t(14)=5.38, p<.001]$ and SPAI $[t(14)=$ $4.99, p<.001]$ from pre to post-assessment, and significant reductions from post-assessment to follow-up on the LSAS [t(14) $=2.47, p=.027]$ and SPAI [ $t(14)=2.43, p=.029]$.

Similarly, a repeated measures ANOVA conducted on the SDS revealed a significant main effect of Time, $\left[F(2,12)=8.38, p=.005, \eta_{p}{ }^{2}=.58\right]$. Follow-up simple contrasts indicated that IMP participants displayed significant reductions in functional interference from pre- to 
post-assessment, $[t(13)=3.95, p=.002]$ and a significant decrease from post- to follow-up assessment, $[t(13)=2.19, p=.048]$. Finally, a repeated measures MANOVA conducted on measures of emotional distress revealed a significant multivariate effect for Time, $[F(4,10)$ $\left.=9.64, p=.002, \eta_{p}{ }^{2}=.79\right]$. Simple follow-up contrasts revealed that the IMP group exhibited significant reductions on symptoms of depression [BDI-II: $t(14)=6.05, p<.001$ ] and general anxiety [STAI-T: $t(14)=5.13, p<.001]$ from pre- to post-assessment; however, scores on the BDI-II and STAI-T did not differ significantly from post-assessment to followup (both $p>.70$ ). Considered together, these results suggest that treatment gains resulting from IMP appear to be an enduring effect, at least during a several month follow-up period.

\section{Responder Status}

Diagnostic status after treatment was examined. At post-assessment, $65 \%$ of participants in the IMP group no longer met diagnostic criteria for GSAD compared to $13 \%$ of participants in the ICC group, $\chi^{2}(1)=12.36, p<.001$. We followed the procedures outlined by Jacobson and Truax (1991) to evaluate clinically significant change on the primary outcome measure (i.e., LSAS). A participant was classified as meeting criteria for clinically significant change if (a) their post-treatment score fell within the range (mean \pm two standard deviations) of the non-clinical population using Fresco et al.'s (2001) non-patient LSAS data $(M=13.61, S D$ $=11.10$; see also Clark et al., 2006), and (b) if they displayed a statistically reliable reduction in scores from pre- to post-assessment using the reliable change index (Jacobson \& Truax, 1991). The percentage of patients who had achieved clinically significant improvement on the LSAS was $30 \%$ in the IMP and $13 \%$ in the ICC, $\chi^{2}(1)=1.86, p=.17$.

\section{Mediation Analyses}

We used bootstrapping procedures (Preacher \& Hayes, 2008) to test the indirect effect of treatment condition (Group: IMP, ICC) on reduction in clinician-rated social anxiety symptoms (LSAS) from pre- to post-assessment through change in interpretation bias. We computed residualized change scores for each measure by regressing the post-treatment scores on the pre-treatment scores for all study participants. We tested a multiple mediator model in which the proposed mediators (i.e., change in threat and benign interpretation bias scores for social and non-social scenarios) were entered simultaneously in the proposed model, which arguably provides a more powerful test of the hypothesis under investigation (cf. separate single mediator models; e.g., Baron \& Kenny, 1986). We present results of the mediation analyses for the $95 \%$ confidence intervals of the indirect path ( $a b)$. Path $a$ represents the direct path from the independent variable (IV) to the putative mediators (M); path $b$ represents the direct path from the mediator to the dependent variable (DV) while the IV is held constant; path $c$ represents the total effect of the IV on the DV; and path $c^{\prime}$ represents the effect of the IV on the DV while controlling for M. Significant indirect effects $(a b)$ are revealed when confidence intervals do not overlap with zero. We report accelerated bias-corrected confidence intervals obtained using 5000 resamples as well as point estimates of the indirect effects.

Results revealed that the overall mediation model accounted for a significant amount of the variance in predicting change in clinician-rated social anxiety symptoms, $R^{2}=.45, F(5,34)$ $=5.66, p<.001$. Only change in social threat interpretation bias predicted change in LSAS when the remaining variables were held constant, with larger reductions in endorsement of threatening interpretations of ambiguous social information associated with a greater decrease in social anxiety symptoms, $B=.74(S E=.22), t(39)=3.32, p=.002$ (path $b 1)$. The indirect effect $(a b)$ of treatment on change in symptoms through change in social threat interpretation bias was significant such that the $95 \%$ confidence interval did not overlap with zero $(a 1 b 1=.5989$; lower value $=.1133$, upper value $=1.3452)$. The direct effect of treatment condition on change in social anxiety remained significant when controlling for 
change in interpretation bias, which suggested partial mediation, $B=.73(S E=.32), t(39)=$ $2.25, p=.03\left(\right.$ path $\left.c^{\prime}\right) .^{5}$

\section{Condition Assignment}

To assess whether participants remained blind to their respective experimental condition, we asked participants at post-assessment whether they thought they received the active intervention or not. Participants were not fully debriefed about the nature of the manipulation prior to providing their condition guess responses. Of the participants who provided responses $(\mathrm{N}=33)^{6}$, in the IMP group $(n=16), 4$ participants thought they received the active treatment. In the ICC group $(n=17), 5$ participants believed they had received the active treatment. Groups did not differ in the proportion of participants who thought they received the active treatment, $\chi^{2}(1)=.081, p=.78$. These findings bolster confidence that participants did not systematically predict their respective experimental condition. An examination of pre- to post-treatment effect sizes on the primary outcome measure (LSAS) revealed that IMP participants displayed treatment changes of similar magnitude regardless of whether they believed they were in the active condition $(d=1.55)$ or not $(d=1.97)$. In contrast, participants in the ICC group who believe they received the active intervention displayed a notably larger treatment response $(d=1.93)$ relative to ICC participants who did not think they were in the active condition $(d=0.41)$.

\section{Discussion}

In the current study we sought to examine the effects of a multi-session computerized interpretation training procedure in a sample of individuals meeting diagnostic criteria for GSAD. Consistent with our prediction, IMP successfully modified interpretations of ambiguous social information as well as reduced symptoms of social anxiety from pre- to post-assessment. Independent assessor ratings at post-assessment indicated that participants who completed IMP were significantly less socially anxious and avoidant as well as less functionally impaired than the control group. Finally, $65 \%$ of participants in the IMP group no longer met DSM-IV criteria for GSAD after training, compared to $13 \%$ of control participants. To our knowledge, the present study provides the first empirical evidence supporting the potential clinical utility of using computerized interpretation training programs alone in individuals diagnosed with GSAD.

Previous studies have demonstrated that computerized CBM paradigms can induce selective interpretive biases of ambiguous material in non-clinical and analogue samples (e.g., Grey \& Mathews, 2000; Mathews \& Mackintosh, 2000; Murphy et al., 2007; Wilson et al., 2006).

However, it is conceivable that interpretation biases are more resistant to change in individuals with more severe and enduring symptoms, in part because they may present with more deeply ingrained negative social beliefs and assumptions. Thus, our first goal was to establish the efficacy of IMP in manipulating the type of social interpretation biases characteristic of individuals with GSAD (e.g., Amir, Foa, \& Coles, 1998; Stopa \& Clark,

\footnotetext{
${ }^{5}$ We conducted a sensitivity analysis using single mediator models in which change in the four interpretation bias scores (i.e., social threat, social benign, general threat, and general benign) were analyzed separately as putative mediators of change in LSAS from preto post-treatment. Consistent with the multiple mediator model reported in the main text, results revealed that only change in social threat interpretation bias was significantly associated with change in LSAS when the remaining variables were held constant, $B=.48$ $(S E=.14), t(39)=3.35, p=.002$ (path $b)$. The indirect effect $(a b)$ of treatment on change in symptoms through change in social threat interpretation bias was significant $(a b=.3841$; lower value $=.0453$, upper value $=.9207)$. Change in social benign interpretation bias was marginally associated with change in LSAS, $B=-.33(S E=.17), t(39)=-1.95, p=.059$ (path $b)$; however, the $95 \%$ confidence interval of the indirect effect was not significant $(a b=.3649$; lower value $=-.0539$, upper value $=.9623)$. Neither change in general threat nor general benign interpretation bias were associated with change in LSAS (both $p>.10$, path $b$ ). These findings are consistent with the multiple mediator analysis reported in the main text.

${ }^{6}$ Only a sub-sample of participants provided a condition assignment response due to an error in the administration of post-assessment debriefing questionnaires that inadvertently neglected to include the measure that assessed belief in condition assignment.
} 
2000; Hirsch \& Mathews, 2000). The present findings demonstrated that relative to a control group that was reinforced equally for making benign versus threat interpretations, IMP resulted in significantly greater pre- to post-treatment reductions in endorsement of threat interpretations and increases in endorsement of benign interpretations of ambiguous social information. Participants were tested on materials at post-assessment that were not encountered during training, suggesting that changes in interpretation associated with IMP generalized to novel stimuli. These results converge with earlier findings in a sample of socially anxious students (Beard \& Amir, 2008) and provide further support for CBM interpretation paradigms that provide individuals with corrective feedback about the way in which they resolve ambiguous scenarios (see also Beard et al., in press; Brosan et al., 2011). The magnitude of change in interpretation bias in the IMP group was large (pre- to postassessment $d s=1.91$ and 1.76 for threat and benign interpretations, respectively) although lower than that observed in the IMP group in Beard and Amir (2008; pre- to post-treatment $d s=2.83$ and 2.71, for threat and benign interpretations, respectively), possibly reflecting differences in severity between the analogue and clinical samples. Nevertheless, the current results support the malleability of interpretive biases using CBM procedures in individuals diagnosed with GSAD.

IMP also modified interpretations of general (non-social) material, producing large effects similar in magnitude to changes in social interpretation bias. These findings are not unexpected given that approximately one-third of IMP trials presented non-social ambiguous scenarios. However, these results raise questions about the optimal dose of training given the relatively robust changes in general threat interpretations with fewer training trials relative to the number of ambiguous social trials. On the one hand, these findings may suggest that fewer trials are needed to shift interpretive biases in anxious individuals. It is also possible that the social interpretation training trials facilitated changes in general interpretations (and vice versa), which would imply that training in one domain generalizes to other domains. Future research is needed to dismantle the relative effects of modifying interpretations of social versus general scenarios in order to determine which type of training materials yield the greatest clinical benefit.

Our second goal was to examine the effects of IMP on social anxiety symptoms and related interference. Participants in the IMP group were judged by independent evaluators as displaying significantly greater reductions in social anxiety symptoms and functional impairment relative to the control group. Thus, the current study adds to a small but growing literature supporting the efficacy of computerized CBM procedures in the treatment of individuals meeting diagnostic criteria for an anxiety disorder (Amir, Beard et al., 2009; Amir, Beard, Taylor et al., 2009; Schmidt et al., 2009). However, whereas previous studies have tested the efficacy of CBM-A procedures alone or in combination with CBM-I procedures in the remediation of anxiety disorders, the current study extends these findings to a program designed to modify interpretive biases characteristic of pathologic anxiety. The robustness of the interpretation training procedure is bolstered by extending earlier findings in analogue samples of socially anxious (Beard \& Amir, 2008) and generally trait anxious individuals (Mathews et al., 2007; Salemink et al., 2009) to clinical samples presenting with greater severity of symptoms and functional interference. It should be noted, however, that the IMP group did not differ significantly from the control group on change in self-rated social anxiety symptoms. Rather, both groups displayed large reductions in self-rated social anxiety symptoms of similar magnitude. This finding points to the potency of the control condition and also suggests that the LSAS was more sensitive to discriminating treatment effects compared to the SPAI.

Follow-up assessments conducted approximately three months post-treatment revealed that IMP participants maintained symptom reduction after completing the training, suggesting 
that the beneficial effects of training were enduring. To our knowledge, this is the first study to examine the longer-term impact of interpretation training procedures. However, follow-up data should be interpreted with caution because of the modest retention rate, the fact that assessors and participants were no longer blind to condition, and the lack of a placebo control group. Future research is needed to investigate the long-term impact of IMP that assesses symptoms as well as interpretation bias.

Participants in the IMP group also displayed significantly greater reductions in self-reported symptoms of depression and trait anxiety relative to the ICC group; although differential group changes on the STAI-T were less robust in the ITT sample. These findings suggest that the effects of IMP generalized to symptoms that often co-occur with social anxiety. It is possible that changes in depression and trait anxiety were simply concomitants of change in social anxiety symptoms (e.g., Moscovitch, Hofmann, Suvak, \& In-Albon, 2005). Another explanation is that IMP modified the types of cognitions that are also hypothesized to be causally implicated in the maintenance of depression and general anxiety (Mathews \& MacLeod, 2005). However, previous findings regarding the effects of CBM interventions on co-occurring symptoms of depression are mixed with some studies finding differential group changes in depression (Amir, Beard et al., 2009) whereas others did not (Amir, Beard, Taylor et al., 2009; Beard \& Amir, 2008; Schmidt et al., 2009). Future research in larger samples is needed to clarify whether CBM procedures that target anxiety-related information processing biases are sufficient to reduce symptoms of depression or whether additional procedures that more directly target depressive cognitions would be beneficial.

How do the present findings compare to extant clinical trials in SAD? First, participants in the current study had clinical characteristics and symptom severity scores in the range of previous treatment studies (e.g., Clark et al., 2006; Davidson et al., 2004; Heimberg et al., 1998). Further, nearly half of participants reportedly sought treatment for their social fears in the past, and over one-quarter were concurrently on medication. Thus, there is little reason to believe that the current sample comprised a less severely anxious or impaired group of individuals. Second, IMP produced treatment effects that were large in magnitude and comparable to those reported in previous studies, suggesting that IMP may be an effective intervention for reducing symptoms of social anxiety. The proportion of participants meeting criteria for clinically significant change was comparable to previous CBM trials (Amir, Beard, Taylor et al., 2009) but lower than that reported in some RCTs of other psychosocial treatments using similar criteria to compute clinical significance (e.g., cognitive therapy; Clark et al., 2006). These findings are particularly remarkable considering that IMP is relatively brief in duration (i.e., 20-minute sessions completed twice weekly for 6 weeks) and was completed in the absence of therapist contact. Thus, IMP may serve as a transportable and widely accessible treatment for individuals with GSAD who are unable to or choose not to access existing treatments (Coles et al., 2004; Huppert et al., 2003; Olfson et al., 2000). Given its relatively straightforward computerized method of delivery, IMP could be implemented within the home or other community-based settings (e.g., primary care). IMP could also be integrated into standard CBT regimens to help facilitate cognitive change during the early stages of treatment or to maintain cognitive changes as part of relapse prevention following treatment.

One unresolved issue involves establishing the optimal dose of CBM-I sessions. The majority of participants in the current study completed 12 training sessions, one-third more than the typical 8 training sessions reported in several previous CBM clinical trials (Amir, Beard et al., 2009; Amir, Beard, Taylor et al., 2009; Beard \& Amir, 2008; Schmidt et al., 2009). Treatment effects obtained in the current study are within the range of effect sizes reported in these previous studies. Moreover, treatment outcomes did not differ significantly in participants who completed the 8 vs. 12-session version of the current protocol. However, 
given the small number of participants who completed the 8 session protocol and site differences on a number of demographics characteristics, these findings are difficult to interpret. Nevertheless, the current findings underscore the potential importance of establishing the number of training sessions required to achieve clinical benefit.

What might account for the reduction in social anxiety associated with IMP? The most parsimonious explanation of the current findings is that IMP changed participants' interpretations of ambiguous social information, which resulted in subsequent reductions in social anxiety symptoms. Consistent with this hypothesis, a mediation analysis revealed that reductions in threatening interpretations of ambiguous social stimuli accounted for changes in social anxiety symptoms. In keeping with findings from previous research (Murphy et al., 2007), participants in the IMP group may have come to view external social situations as less threatening. In turn, diminished threat appraisals may lead to fewer avoidance behaviors, and may have provided IMP participants with greater opportunities to participate in and more objectively evaluate social situations, resulting in reductions in anxiety symptoms. Changes in interpretation bias may have also facilitated more accurate processing of social information that could be used to disconfirm the individual's negative beliefs (e.g., Clark \& Wells, 1995; Hofmann, 2007). Results of the mediation analysis should be interpreted with caution, however, given that temporal precedence of change in the mediator and clinical improvement was not established. Thus, although causal inferences can be made about change in interpretations resulting from the IMP, we cannot make such claims about the relationship between change in interpretations and symptom change. Future research should administer assessments of interpretation bias at multiple points during the course of treatment to better address these issues. It should also be noted that although change in threat interpretation bias was the strongest mediator of change in symptoms in the current study, change in benign interpretation bias was a stronger mediator of treatment response in a previous CBM-I trial (Beard \& Amir, 2008). Although differences in sample characteristics (e.g., analogue vs. clinical) or assessments used in the mediation analysis (e.g., self- vs. clinician-rated symptoms) may account in part for these discrepancies, a true dismantling study that examines the independent effects of targeting threat versus benign interpretation biases is needed to reconcile the relative importance of each process in the treatment of GSAD.

Contemporary cognitive and behavioral treatment regimens for GSAD target relatively explicit cognitive biases. For example, patients may be asked to identify the type of cognitive error ascribed to a particular thought and then to create a rationale response to dispute the distortion (e.g., Davidson et al., 2004). In contrast, participants in the current study were not given a rationale indicating that the program was designed to modify negative biases in thinking. Moreover, the majority of participants were unaware of their condition assignment. One interpretation of those findings is that IMP altered interpretations at a more implicit level relative to interventions that seek to explicitly draw a connection between negative biases in thinking and maintenance of symptoms. However, the current study did not formally assess whether participants were aware that the program was teaching them to make less negative and more benign interpretations of ambiguous situations. Future studies should establish whether change in interpretations occurs more at an implicit versus explicit level in IMP as well as the relative efficacy of procedures that target biases at each level of processing. It may also be beneficial to provide a rationale for the training procedures and to more explicitly encourage participants to generate a link between the sentence and word in relation to themselves during training.

It is notable that the placebo control group (ICC) also showed significant and medium to large changes on most outcome assessments. What might account for these changes? First, ICC participants were reinforced during half of the training trials for making less threatening 
and more benign interpretations. Thus, for participants whose natural tendency at preassessment was to endorse threat and reject benign interpretations, the 50-50 feedback manipulation may have acted similar to a diluted version of IMP. It is also possible that the ICC, by requiring participants to actively think about the relationship between ambiguous social scenarios and emotionally valenced outcomes, increased participants' awareness of their habitual thinking patterns. This heightened awareness may have incidentally led some participants to be more critical of their negative thoughts in social situations, which is consistent with comments from several ICC participants during the post-assessment debriefing. It is also possible that demand effects at least in part influenced changes in the ICC group. Several participants in the ICC group believed they received the active intervention. The magnitude of treatment effects was four times as large in the sub-group of ICC participants who believed they received the active intervention versus those who did not. Symptom reduction in the ICC group may also reflect a non-specific response to receiving attention from an experimenter on multiple occasions. Research is needed to test the effects of alternate placebo conditions (e.g., no feedback condition) that may serve as better experimental controls.

Our study has limitations. First, our sample size was small. This provided limited power to conduct mediation analysis and prevented an examination of moderators of treatment response. Second, we did not include an independent measure of interpretation bias that differed from the task used during training. Thus, it is not clear whether participants simply learned a rule that they applied to the specific task or whether IMP changed interpretation processes at a more fundamental level. For example, previous studies that have included independent measures of interpretation do not always find that changes in interpretation on the training task generalized to a different task (e.g., Salemink et al., 2007). Nevertheless, changes in interpretation as measured by the WSAP in the current study were associated with clinical improvement. Future research should also include measures of other cognitive biases (e.g., attention) as well as behavioral tests (e.g., speech task) immediately after training to more clearly establish the mechanism of action and generalizability of treatment effects. Another limitation is that we did not comprehensively assess for comorbid psychiatric conditions. Thus, we could not examine whether comorbidity moderated the effects of IMP or whether IMP also affected diagnostic status for co-occurring conditions. It should also be noted that treatment completion rates may have been influenced by providing monetary compensation to participants for attending treatment sessions (see Beard et al., in press).

Given that participants were aware at the outset of the study that they could be assigned to a condition designed to reduce social anxiety, the possibility of demand effects should also be considered. However, we believe that demand effects are unlikely to explain the observed pattern of findings. First, groups did not differ in the proportion of participants who believed they received the active intervention, suggesting that differential group outcomes could not be attributed to demand alone. Second, the magnitude of treatment effects was similar in IMP participants who believed they received the active treatment versus those who did not. Nevertheless, future research should include behavioral (or other) assessments that are less susceptible to experimental demand.

In summary, the current findings provide initial support for the efficacy of computerized interpretation training procedures in individuals diagnosed with GSAD. In doing so, this study extends previous CBM research in clinical populations that have demonstrated the efficacy of attentional training manipulations (Amir, Beard et al., 2009; Amir, Beard, Taylor et al., 2009; Schmidt et al., 2009) or combination CBM-A and CBM-I procedures (Beard et al., in press; Brosan et al., 2011). Future studies should examine the additive and/or interactive effects of CBM procedures that target attention and interpretation biases 
characteristic of anxiety (e.g., Broson et al., 2011). Similarly, given that interpretation biases are key targets of cognitive and behavioral interventions for GSAD, it may be worthwhile to examine whether IMP can enhance or accelerate cognitive changes during exposure-based treatments. A large randomized clinical trial comparing IMP to established treatments (e.g., CBT) as well as their combination will help further establish the clinical utility of computerized interpretation training programs.

\section{Acknowledgments}

This research was supported by grants from the National Institutes of Health awarded to the first author (R34MH073004-01, R34MH077129-01, R01MH087623-01) and second author (K99MH090243-01). We would like to thank Sadia Najmi, Courtney Beard, Jessica Bomyea, Amanda Morrison, and Christal Badour for their help with data collection and management.

\section{References}

Amir N, Beard C, Burns M, Bomyea J. Attention modification program in individuals with Generalized Anxiety Disorder. Journal of Abnormal Psychology. 2009; 118:28-33. [PubMed: 19222311]

Amir N, Beard C, Taylor CT, Klumpp H, Elias J, Burns M, Chen X. Attention training in individuals with generalized social phobia: A randomized controlled trial. Journal of Consulting and Clinical Psychology. 2009; 77:961-973. [PubMed: 19803575]

Amir N, Foa EB, Coles ME. Negative interpretation bias in social phobia. Behaviour Research and Therapy. 1998; 36:945-958. [PubMed: 9714945]

Amir N, Weber G, Beard C, Bomyea J, Taylor CT. The effect of a single session attention modification program on response to a public speaking challenge in socially anxious individuals. Journal of Abnormal Psychology. 2008; 117:860-868. [PubMed: 19025232]

Baron RM, Kenny DA. The moderator-mediator distinction in social psychological research: Conceptual, strategic and statistical considerations. Journal of Personality and Social Psychology. 1986; 51:1173-1182. [PubMed: 3806354]

Beard C. Cognitive bias modification for anxiety: Current evidence and future directions. Expert Review of Neurotherapeutics. 2011; 11:299-311. [PubMed: 21306216]

Beard C, Amir N. A multi-session interpretation modification program: Changes in interpretation and social anxiety symptoms. Behaviour Research and Therapy. 2008; 46:1135-1141. [PubMed: 18675400]

Beard C, Amir N. Interpretation in social anxiety: When meaning precedes ambiguity. Cognitive Therapy and Research. 2009; 33:406-415. [PubMed: 20046862]

Beard C, Amir N. Negative interpretation bias mediates the effect of social anxiety on state anxiety. Cognitive Therapy and Research. 2010; 34:292-296. [PubMed: 20495620]

Beard C, Weisberg RB, Amir N. Combined cognitive bias modification treatment for social anxiety disorder: A pilot study. Depression and Anxiety. (in press).

Beck, AT.; Steer, RA.; Brown, GK. Manual for Beck Depression Inventory-II. San Antonio, TX: Psychological Corporation; 1996.

Brosan L, Hoppitt L, Shelfer L, Sillence A, Mackintosh B. Cognitive bias modification for attention and interpretation reduces trait and state anxiety in anxious patients referred to an out-patient service: Results from a pilot study. Journal of Behavior Therapy and Experimental Psychiatry. 2011; 42:258-264. [PubMed: 21349242]

Clark DM, Ehlers A, Hackmann A, McManus F, Fennell M, Grey N, et al. Cognitive therapy versus exposure and applied relaxation in social phobia: A randomized controlled trial. Journal of Consulting and Clinical Psychology. 2006; 74:568-578. [PubMed: 16822113]

Clark, DM.; Wells, A. A cognitive model of social phobia. In: Heimberg, RG.; Liebowitz, MR.; Hope, DA.; Schneier, FR., editors. Social phobia: Diagnosis, assessment, and treatment. New York: Guilford Press; 1995. p. 69-93. 
Coles ME, Turk CL, Jindra L, Heimberg RG. The path from initial inquiry to initiation of treatment of social anxiety disorder in an anxiety disorders specialty clinic. Journal of Anxiety Disorders. 2004; 18:371-383. [PubMed: 15125983]

Constans J, Penn D, Ihen G, Hope D. Interpretive biases for ambiguous stimuli in social anxiety. Behaviour Research and Therapy. 1999; 37:643-651. [PubMed: 10402689]

Davidson JR, Foa EB, Huppert JD, Keefe FJ, Franklin ME, Compton JS, et al. Fluoxetine, comprehensive cognitive behavioral therapy, and placebo in generalized social phobia. Archives of General Psychiatry. 2004; 61:1005-1013. [PubMed: 15466674]

First, MB.; Spitzer, RL.; Gibbon, M.; Williams, JBW. Structured Clinical Interview for Axis I DSMIV Disorders-Patient Edition. New York: Biometrics Research Department; 1994.

Fresco DM, Coles ME, Heimberg RG, Liebowitz MR, Hami S, Stein MB, et al. The Liebowitz Social Anxiety Scale: A comparison of the psychometric properties of self-report and clinicianadministered formats. Psychological Medicine. 2001; 31:1025-1035. [PubMed: 11513370]

Grey S, Mathews A. Effects of training on interpretation of emotional ambiguity. The Quarterly Journal of Experimental Psychology. 2000; 53A:1143-1162. [PubMed: 11131817]

Hambrick JP, Turk CL, Heimberg RG, Schneier FR, Liebowitz MR. Psychometric properties of disability measures among patients with social anxiety disorder. Journal of Anxiety Disorders. 2004; 18:825-839. [PubMed: 15474855]

Hayes S, Hirsch CR, Krebs G, Mathews A. The effects of modifying interpretation bias on worry in generalized anxiety disorder. Behaviour Research and Therapy. 2010; 48:171-178. [PubMed: 19857859]

Heimberg RG, Horner KJ, Juster HR, Safren SA, Brown EJ, Schneier FR, Liebowitz MR. Psychometric properties of the Liebowitz Social Anxiety Scale. Psychological Medicine. 1999; 29:199-212. [PubMed: 10077308]

Heimberg RG, Liebowitz MR, Hope DA, Schneier FR, Holt CS, Welkowitz LA, et al. Cognitive behavioral group therapy vs. Phenelzine therapy for social phobia. Archives of General Psychiatry. 1998; 55:1133-1141. [PubMed: 9862558]

Hirsch C, Mathews A. Impaired positive inferential bias in social phobia. Journal of Abnormal Psychology. 2000; 109:705-712. [PubMed: 11195994]

Hofmann SG. Cognitive factors that maintain social anxiety disorder: A comprehensive model and its treatment implications. Cognitive Behaviour Therapy. 2007; 36:195-209.

Hofmann S, Smits J. Cognitive-behavioral therapy for adult anxiety disorders: A meta-analysis of randomized placebo-controlled trials. Journal of Clinical Psychiatry. 2008; 69:621-632. [PubMed: 18363421]

Huppert JD, Foa E, Fur JM, Filip JC, Mathews A. Interpretation bias in social anxiety: A dimensional perspective. Cognitive Therapy and Research. 2003; 27:569-577.

Huppert JD, Franklin ME, Foa EB, Davidson JRT. Study refusal and exclusion from a randomized treatment study of generalized social phobia. Journal of Anxiety Disorders. 2003; 17:683-693. [PubMed: 14624818]

Jacobson NS, Truax P. Clinical significance: A statistical approach to defining meaningful change in psychotherapy research. Journal of Consulting and Clinical Psychology. 1991; 59:12-19. [PubMed: 2002127]

Kessler RC, Berglund P, Demler O, Jin R, Walters EE. Lifetime prevalence and age-of-onset disturbances of DSM-IV Disorders in the National Comorbidity Survey Replication. Archives of General Psychiatry. 2005; 62:593-602. [PubMed: 15939837]

Koster EHW, Fox E, MacLeod C. Introduction to the special section on cognitive bias modification in emotional disorders. Journal of Abnormal Psychology. 2009; 118:1-4. [PubMed: 19222308]

Liebowitz MR. Social phobia. Modern Problems of Pharmacopsychiatry. 1987; 22:141-173. [PubMed: 2885745]

Leon AC, Olfson M, Portera L, Farber L, Sheehan DV. Assessing psychiatric impairment in primary care with the Sheehan Disability Scale. International Journal of Psychiatry Medicine. 1997; 27:93105.

MacLeod C, Rutherford E, Campbell L, Ebsworthy G, Holker L. Selective attention and emotional vulnerability: Assessing the causal basis of their association through the experimental 
manipulation of attentional bias. Journal of Abnormal Psychology. 2002; 111:107-123. [PubMed: 11866165]

Mathews A, Macintosh B. Induced emotional interpretation bias and anxiety. Journal of Abnormal Psychology. 2000; 109:602-615. [PubMed: 11195984]

Mathews A, MacLeod C. Cognitive vulnerability to emotional disorders. Annual Review of Clinical Psychology. 2005; 1:197-225.

Mathews A, Ridgeway V, Cook E, Yiend J. Inducing a benign interpretational bias reduces trait anxiety. Journal of Behavior Therapy and Experimental Psychiatry. 2007; 38:225-236. [PubMed: 17141179]

Mennin DS, Fresco DM, Heimberg RG, Schneier FR, Davies SO, Liebowitz MR. Screening for social anxiety disorder in the clinical setting: Using the Liebowitz Social Anxiety Scale. Journal of Anxiety Disorders. 2002; 16:661-673. [PubMed: 12405524]

Moser JS, Hajcak G, Huppert JD, Foa EB, Simons RF. Interpretation bias in social anxiety as detected by event-related brain potentials. Emotion. 2008; 8:693-700. [PubMed: 18837619]

Moscovitch DA, Hofmann SG, Suvak MK, In-Albon T. Mediation of changes in anxiety and depression during treatment of social phobia. Journal of Consulting and Clinical Psychology. 2005; 73:945-952. [PubMed: 16287394]

Murphy R, Hirsch CR, Mathews A, Smith K, Clark DM. Facilitating a benign interpretation bias in a high socially anxious population. Behaviour Research and Therapy. 2007; 45:1517-1529. [PubMed: 17349970]

Olfson M, Guardino M, Streuning E, Scheier R, Hellman F, Klein DF. Barriers to the treatment of social anxiety. American Journal of Psychiatry. 2000; 157:521-527. [PubMed: 10739410]

Preacher KJ, Hayes AF. Asymptotic and resampling strategies for assessing and comparing indirect effects in multiple mediator models. Behavior Research Methods. 2008; 40:879-891. [PubMed: 18697684]

Rapee RM, Heimberg RG. A cognitive-behavioral model of anxiety in social phobia. Behaviour Research and Therapy. 1997; 35:741-756. [PubMed: 9256517]

Schmidt NB, Richey JA, Buckner JD, Timpano KR. Attention training for generalized social anxiety disorder. Journal of Abnormal Psychology. 2009; 118:5-14. [PubMed: 19222309]

Salemink E, van den Hout M, Kindt M. Trained interpretive bias: Validity and effects on anxiety. Journal of Behavior Therapy and Experimental Psychiatry. 2007; 38:212-224. [PubMed: 17141735]

Salemink E, van den Hout M, Kindt M. Effects of positive interpretive bias modification in highly anxious individuals. Journal of Anxiety Disorders. 2009; 23:676-683. [PubMed: 19272750]

Spielberger, CD.; Gorsuch, RL.; Lushene, R.; Vagg, PR.; Jacobs, GA. Manual for the State-Trait Anxiety Inventory. Palo Alto, CA: Consulting Psychologist Press; 1983.

Stein MB, Stein DJ. Social Anxiety Disorder. Lancet. 2008; 371:1115-1125. [PubMed: 18374843]

Stopa L, Clark D. Social phobia and interpretation of social events. Behaviour Research and Therapy. 2000; 38:273-283. [PubMed: 10665160]

Turner SM, Beidel DC, Dancu CV, Stanley MA. An empirically derived inventory to measure social fears and anxiety: The Social Phobia and Anxiety Inventory. Psychological Assessment. 1989; $1: 35-40$.

Wilson E, MacLeod C, Mathews A, Rutherford E. The causal role of interpretive bias in anxiety reactivity. Journal of Abnormal Psychology. 2006; 115:103-111. [PubMed: 16492101] 


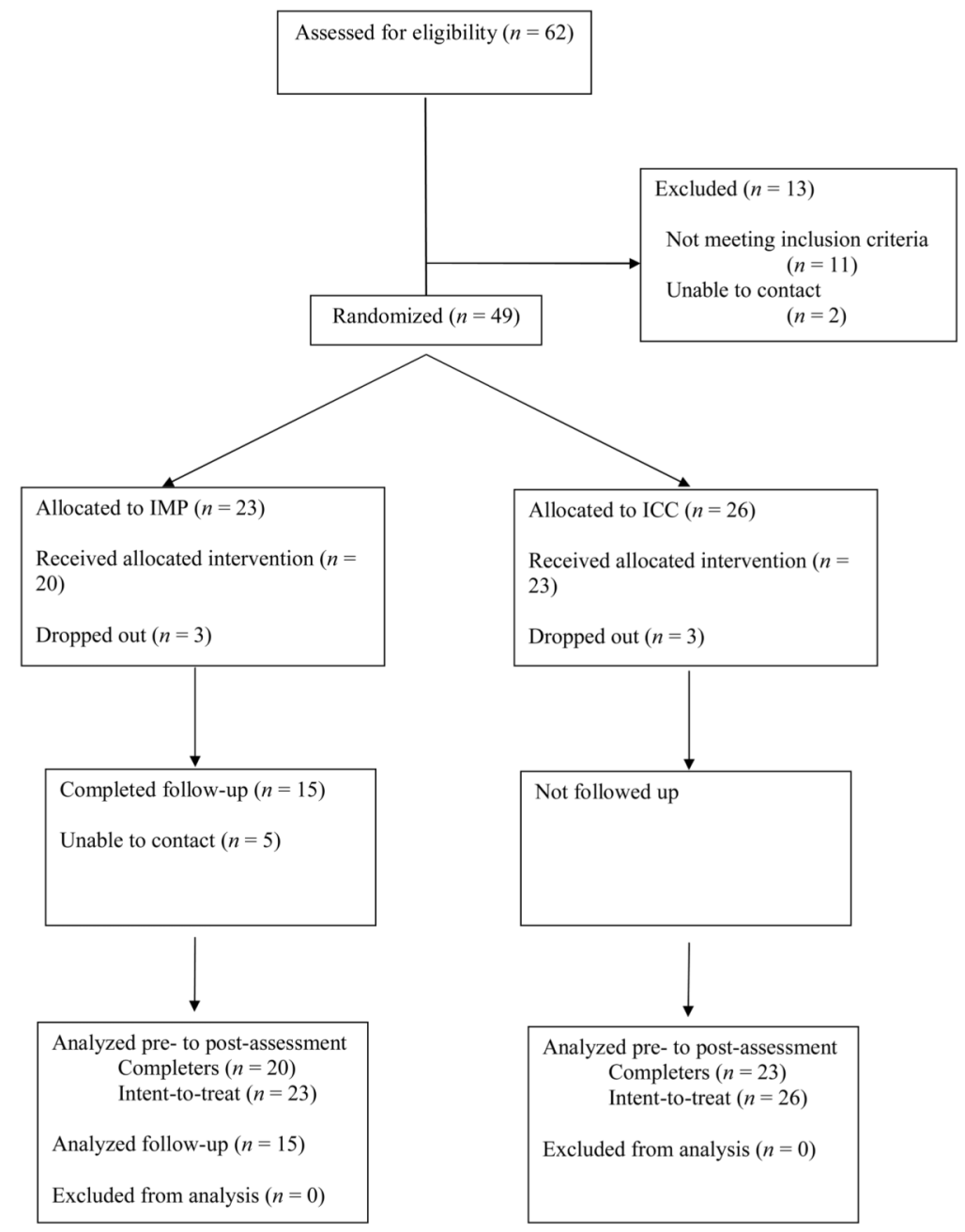

Figure 1.

Diagram of participants' progress through phases of the study. 
Table 1

Patient Demographics and Clinical Characteristics

\begin{tabular}{lcc}
\hline Variable & IMP $(\boldsymbol{n}=\mathbf{2 3})$ & ICC $(\boldsymbol{n}=\mathbf{2 6})$ \\
\hline Gender (\% female) & 82.6 & 61.5 \\
Age & $28.9(11.2)$ & $33.1(12.9)$ \\
Years of Education & $13.1(5.4)$ & $15.0(2.2)$ \\
Ethnicity (\%) & & \\
Caucasian & 34.8 & 57.7 \\
Asian American & 8.7 & 11.5 \\
African American & 13.0 & 3.8 \\
Latin American & 8.7 & 15.4 \\
Bi-racial & 13.0 & 3.8 \\
Other & 17.4 & 7.7 \\
Relationship Status (\%) & & \\
Married/Common Law & 8.7 & 23.1 \\
Divorced/Separated & 13.0 & 3.8 \\
Never Married & 78.3 & 73.1 \\
Current Mediation Use $(\%)$ & 27.3 & 28.0 \\
Past Treatment for SAD $(\%)$ & 43.5 & 57.7 \\
\hline
\end{tabular}

Note. Standard deviations in parentheses. $\mathrm{SAD}=$ social anxiety disorder. 


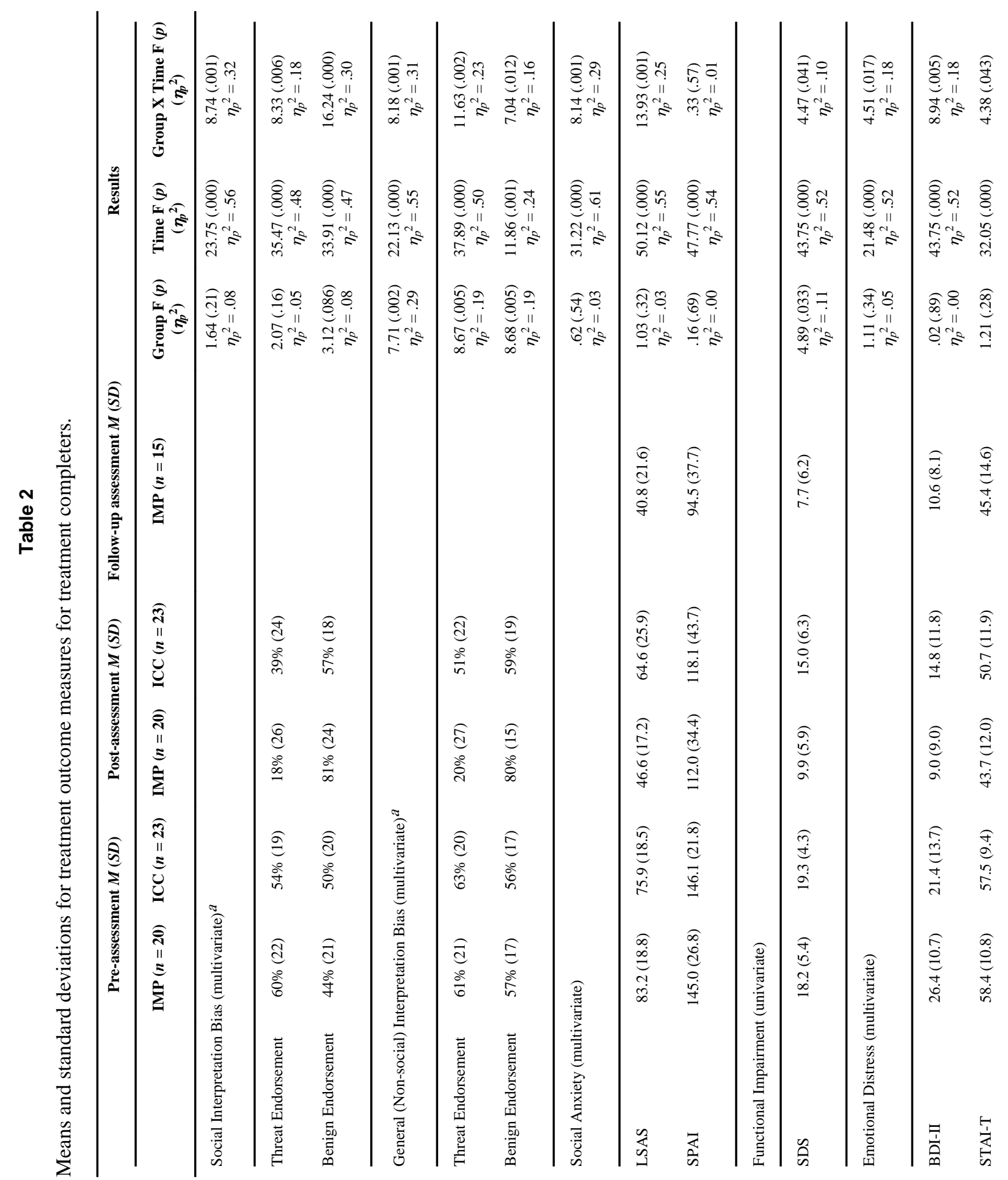




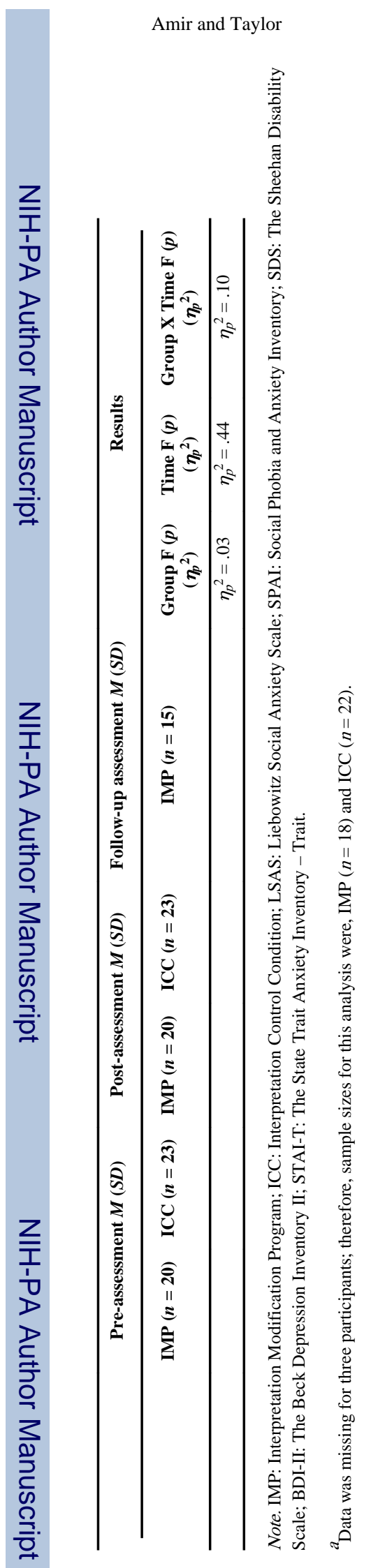

Page 23 
Table 3

Effect sizes for the treatment outcome variables for treatment completers.

\begin{tabular}{llll}
\hline & \multicolumn{3}{c}{ Effect size } \\
\cline { 2 - 3 } Variable & Within-group $\boldsymbol{a}$ & \\
\cline { 2 - 3 } & IMP & ICC & Between-group $\boldsymbol{b}$ \\
\hline Social Interpretation Bias & & & \\
Threat Endorsement & 1.91 & 0.79 & 0.92 \\
Benign Endorsement & 1.76 & 0.35 & 1.30 \\
\hline General (Non-social) Interpretation Bias & \\
Threat Endorsement & 1.97 & 0.59 & 1.29 \\
Benign Endorsement & 1.39 & 0.17 & 1.22 \\
\hline Social Anxiety & & & \\
LSAS & 1.95 & 0.61 & 1.05 \\
SPAI & 1.23 & 1.29 & 0.17 \\
\hline Functional Impairment & & & \\
SDS & 1.52 & 0.99 & 0.80 \\
\hline Emotional Distress & & & 0.83 \\
BDI-II & 1.62 & 0.48 & 0.66 \\
STAI-T & 1.35 & 0.72 & \\
\hline
\end{tabular}

Note. IMP: Interpretation Modification Program; ICC: Interpretation Control Condition; LSAS: Liebowitz Social Anxiety Scale; SPAI: Social Phobia and Anxiety Inventory; SDS: The Sheehan Disability Scale; BDI-II: The Beck Depression Inventory II; STAI-T: The State Trait Anxiety Inventory - Trait.

${ }^{a}$ Within group pre- to post-treatment effect sizes $=[($ pre-treatment mean - post-treatment mean $) /$ pre-treatment standard deviation $\left.)\right]$.

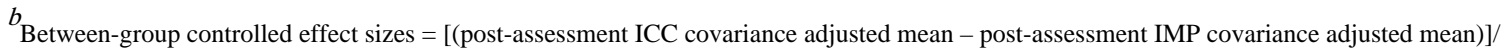
pooled standard deviation. 


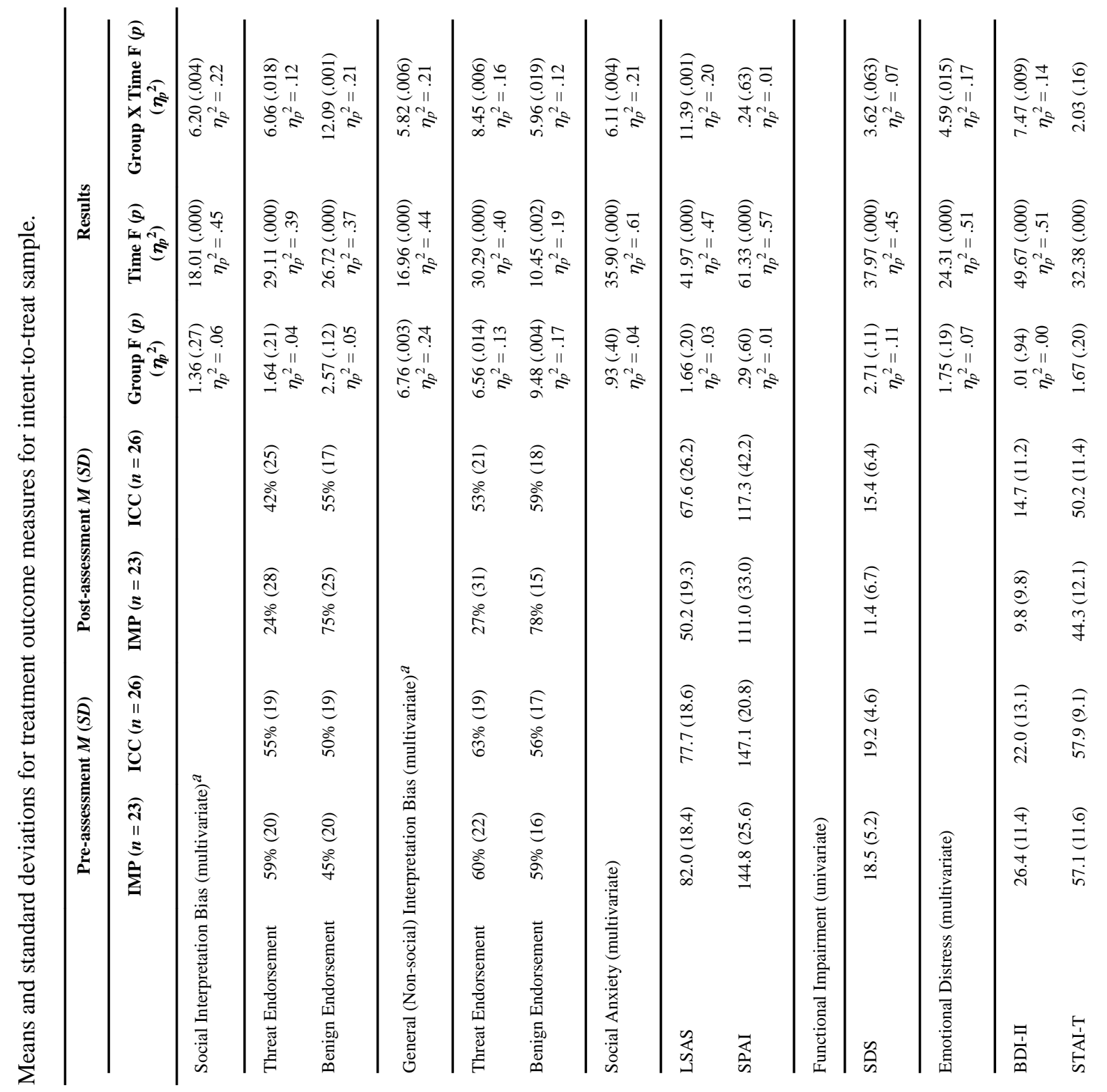




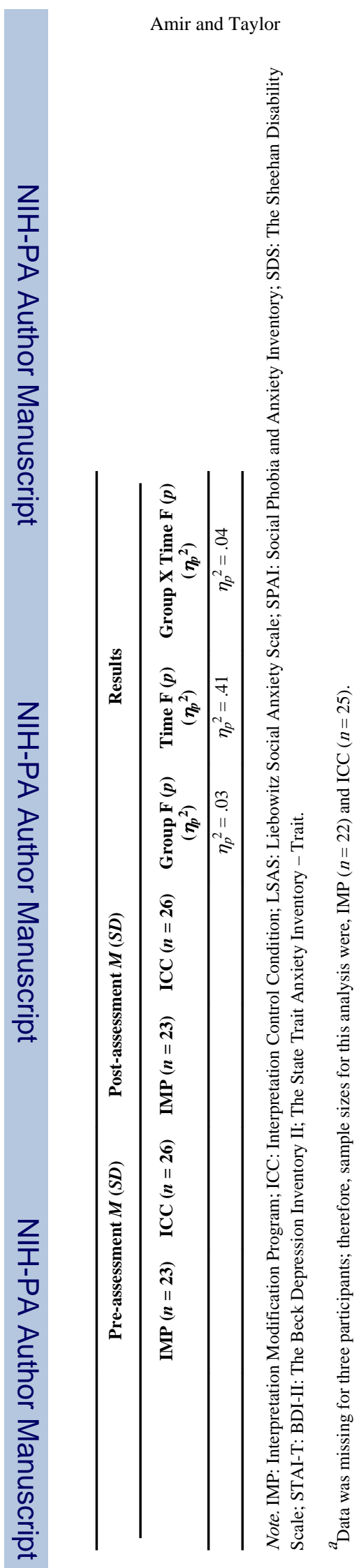

Page 26 


\section{Appendix}

Sample interpretation assessment and training materials.

\begin{tabular}{llll}
\hline & Threat word & Benign word & Ambiguous sentence \\
\hline Social & & & \\
& Criticize & Praise & Your boss wants to meet with you. \\
& Embarrassing & Funny & People laugh after something you said. \\
& Mad & Distracted & A friend does not respond when you wave hello. \\
& Ugly & Attractive & An old friend comments on how you look different now. \\
\hline Non-social & Tumor & Height & The doctor examined your growth. \\
& Broke & Wealthy & Your bank statement is surprising. \\
& Panicky & Exercise & Your heart is beating very quickly. \\
& Sick & Hot & Your face feels sweaty. \\
\hline
\end{tabular}

